Methods In August 2013 an electronic survey containing 20 questions was sent to the $18 \mathrm{RCPCH}$-approved National GRID paediatric gastroenterology training centres who also act as regional referral centres. The questionnaire was completed online by either the departmental clinical or endoscopy lead and results collated by the study centre. Descriptive statistics were used to present results.

Results Sixteen centres responded, representing the experience of 65.6 whole-time equivalent (WTE) consultant paediatric gastroenterologists. Half of centres provided out-of-hours cover for GI emergencies in their region, with surgeons providing the majority (69\%) of out-of-hours support, often in conjunction with GI specialists (44\%), for acute upper GI bleeds. 11/16 centres dealt with $<20$ endoscopies for upper GI bleeding annually with 3 centres dealing with $>40 /$ year. $63 \%$ of tertiary centres had a GI haemorrhage protocol available in electronic format (online/shared-drive), but $53 \%$ were not aware of a similar protocol in their respective DGHs; only $31 \%$ of centres provided 'at-risk' patients with emergency cards/advice. $88 \%$ of centres had access to interventional radiology in-hours and 63\% out-of-hours. The injection of vasoconstrictors/sclerosants/thrombotic agents and endoclips were available in all but one centre, however only $19 \%$ of centres (with between 3-4.6 WTE consultants) stated that all their consultants were competent in managing upper GI bleeds, with the same percentage stating that no consultant was competent in management; very few procedures were carried out by trainees. Only half of centres were undertaking regular case review of paediatric bleeding cases. All respondents were keen to be involved in a detailed review of UK practice.

Conclusion Our national survey of tertiary paediatric GI unit experiences' of acute upper GI bleeding demonstrates that a large proportion of centres do not have protocols in place in their own centre or referring units, with most centres performing fewer than 20 therapeutic endoscopic procedures for upper GI bleeding annually. The majority of centres have a small number of consultants competent in upper GI haemorrhage management with limited opportunities for training. There is also limited development of managed clinical networks that would allow more ready access to expert endoscopy provision.

Disclosure of Interest None Declared.

\section{PTH-062 EMERGENCY ADMISSIONS FOR ALCOHOL RELATED CONDITIONS: MAKING SENSE OF ROUTINE DATA}

${ }^{1} \mathrm{P}$ Lekharaju*, ${ }^{2} \mathrm{E}$ Thompson, ${ }^{3} \mathrm{M}$ Shawihdi, ${ }^{2} \mathrm{M}$ Pearson, ${ }^{1} \mathrm{~S}$ Hood, ${ }^{3} \mathrm{~K}$ Bodger. ${ }^{1}$ Dept of Gastroenterology, Aintree University Hospital, University of Liverpool, Liverpool, UK; ${ }^{2}$ Aintree Health Outcome Partnership, University of Liverpool, Liverpool, UK; ${ }^{3}$ Department of Gastroenterology, Institute of Translational Medicine, University of Liverpool, Liverpool, UK

\subsection{6/gutjnl-2014-307263.508}

Introduction Alcohol-related emergency admissions (ARA) are a major and rising hospital burden, resulting from conditions that range from short-term toxicity to end-stage organ damage, notably liver disease. We report a project to develop analyses of routine coding data, with a particular focus on metrics related to 'frequent flyers' (FFs) as targets for new service interventions.

Methods Sources of data: Hospital Episode Statistics for all English hospitals (2006-2008); Inpatient coding data and AED attendances for our Trust (2006-2013).

Analysis: Screening of all non-emergency episodes for alcohol-related codes at any position to flag ARAs; linkage of individual cases to extract all admissions and order chronologically; allocation of each admission to a category based on primary and lower order diagnoses (flagging LIVER and NON-LIVER admissions); identification of frequent flyers (FFs) based on various definitions of admission count; linkage of ARAs to all-cause AED attendances in local data; funnel-plot analyses of patterns across English Trusts; longitudinal trends in local data.

Results Nationally: 219,158 ARAs in 139,077 patients (20062008), mean age (sd): 49.5 yrs (16); males: 99,271 (71\%); Deprivation: Quintile 1 (most deprived) 36.4\%, Quintile 5 (least): 9.4\%; Co-morbidity (Charlson), 0.44 (0.68); LoS: 7 (14) days; Inpatient death: 6,656 (4.8\%). No. admissions ranged from 160 per individual. Frequent Flyers: In two years, a cut-off of $5+$ admissions identified 5,404 FFs (4\% of patients; accounting for $18 \%$ of ARAs nationally) whereas $10+$ identified 909 FFs (1\% of patients; $6 \%$ of ARAs). Mean ARAs per Trust was 927 (range: $235-3930)$ with 6 -fold variation in $\%$ of FFs (1.3\% to $7.7 \%)$ and 4-fold variation in \% with liver disease across English hospitals (range: 7.6-30.2\%). As expected, FFs coded with liver disease had significantly higher LoS and mortality risk consistent with end-stage organ damage and "unavoidable" admissions.

Locally: 21,308 ARAs in 16,305 patients (2006-2012), with annual number of cases rising from 1,615 to 4,603. Defining FFs as $5+$ ARAs per year, there were 320 FFs (2\% of patients; $10 \%$ of ARAs). There was a year-on-year rise in ARAs $(2,454$ $5,510)$ and AED attendances without admission $(2,499-5,979)$. However, FFs ( $5+$ admissions) declined from 64 to 47 between 2006 and 2012 and non-liver FFs from 25 to 12, suggesting a positive impact of new local services on multiple attenders, especially those lacking established liver disease.

Conclusion We have developed a set of candidate metrics focussed on FFs, short stays and selected baskets of conditions to provide data to support front-line acute alcohol services.

Disclosure of Interest None Declared.

\section{PTH-063 IS EVENING ENDOSCOPY COST EFFECTIVE?}

P Closier*, C Hollywood. Gloucestershire Royal Hospital, Gloucester, UK

\subsection{6/gutjnl-2014-307263.509}

Introduction Currently in the UK we are seeing an increasing demand for endoscopy services that is set to continue to rise over the coming years. ${ }^{1}$ Our aim as an endoscopy department is to deliver a patient focused service, and patient choice is an integral component of this. The option of evening endoscopy lists is a novel approach to widening patient choice for provided services whilst simultaneously working towards a 7 day working week. Our aim was to evaluate the success of evening lists in terms of attendance and endoscopic outcomes to ensure the most cost effective evening service whilst identifying potential areas for development.

Methods We analysed data for scheduled evening endoscopy sessions (18.00 to 21.00) booked at Gloucestershire Royal Hospital over a 3 month period from April 2013. Information about preendoscopy fast, endoscopic results and non-attendance (DNA) rates was processed.

Results 54 patients were listed for endoscopy, of these 18 (33\%) endoscopies were inconclusive; 6 (35\%) abandoned due to food bolus, with 9 (53\%) non-attenders. Food bolus patients had fasting times between 6-8 $\mathrm{h}$ (as per protocol), but all had taken a substantial meal pre-fasting and 50\% were diabetic. All nonattenders were booked for variceal surveillance.

Conclusion Developing evening services is essential to meet user needs; however more guidance is needed to ensure cost effectiveness for the trust. In order to run the most efficient service 
we need a more specific target audience, excluding certain patient groups (variceal surveillance and diabetics) and prioritising others (2 week waits). Variceal surveillance patients had high DNA rates and would be more appropriate on a daytime list where a DNA can be replaced by an inpatient. Diabetics would benefit from morning lists, both for easier glycaemic control and to reduce food bolus (with a longer overnight fast). Improved patient fasting information is essential with specific guidance for pre-fasting meals, with focus on reducing 'heavy' foods, particularly rice based meals. Further work is needed to identify other areas for improvement and refine the service, and ultimately produce a protocol for evening endoscopy that is generalisable to UK endoscopy departments.

\section{REFERENCE}

1 NHS Improvement. Rapid Review of Endoscopy Services, March 2012; www. improvement.nhs.uk

Disclosure of Interest None Declared.

\section{PTH-064 ARE OUTCOMES FOLLOWING ENDOSCOPY FOR EMERGENCY UPPER GI BLEEDING WORSE AT NIGHT AND WEEKENDS?}

A Rehman, P Mundre*, B Rembacken. Gastroenterology, Leeds Institute of Gastroenterology, St James S University Hospital, Leeds, UK

\subsection{6/gutjpl-2014-307263.510}

Introduction Patients admitted out of hours or at weekends may have an excess mortality due to the fact that they undergo emergency endoscopy by junior staff. We retrospectively looked at the predictors of mortality in patients undergoing emergency endoscopy for severe bleeding in Leeds.

Methods The survival of patients with the most significant upper GI bleeding lesions who underwent emergency endoscopy in Leeds between end of April 2008 and middle of December 2011 were selected for retrospective analysis using data from our endoscopy reporting system and hospital records.

Results A total of 1663 emergency endoscopies were carried out during this period. From this number, 390 examinations (23\% of total) were selected for more careful scrutiny as the following significant bleeding lesions had been recorded; 136 duodenal ulcers, 84 gastric ulcers, 134 oesophageal varices, 25 gastric varices, 11 Dieulafoy's and 9 other bleeding lesions.

After $23 \%$ of procedures (91/390), the patient died within 30 days. As expected, patients who died had a significantly higher
Rockall score (7.5 vs. 5.9 p $<0.0001)$, a higher ASA level $(3.5$ vs. $2.8 \mathrm{p}<0.001)$ and a lower systolic BP at the time of the examination (95 vs. $102 \mathrm{p}=0.025$ ). Patients who died following endoscopy for bleeding ulcers were significantly older than those who survived (76.6 vs. 67.2 yrs, $\mathrm{p}=0.006$ ). There was no significant difference in mortality with the type of bleeding lesion, $\mathrm{Hb}$ (7.9 vs. 8.0$)$ or heart rate (100 vs. $102 \mathrm{bpm})$ at the time of the endoscopy between those who survived and those who died.

Furthermore, undergoing an emergency gastroscopy at night or during the weekend or bank holiday was not associated with an increased risk of death $(\mathrm{P}=0.24$ and $\mathrm{p}=0.53$ respectively).

Whether the examination was carried out by an $S p R$ or a Consultant made no difference to the survival of the patient. The only link between endoscopic intervention and patient outcome was the finding that patients with varices requiring balloon tamponade where significantly more likely to die (15/36 vs. $16 /$ $98 \mathrm{p}=0.01$ ).

Conclusion Our study had the statistical power to detect all the recognised risk factors for death following admission with an acute upper GI bleed including advancing age, increasing comorbidity and hypotension. We found no evidence that undergoing an emergency endoscopy at night or during the weekend or a bank holiday had any adverse effect on outcomes. Similarly, the level of seniority of the endoscopist did not affect outcomes. Disclosure of Interest None Declared.

\section{PTH-065 IMPACT OF A STANDARDISED CLINICAL MANAGEMENT NETWORK FOR COMPLEX POLYPS WITHIN THE BOWEL CANCER SCREENING PROGRAMME (BCSP)}

${ }^{1} \mathrm{R}$ Ramaraj*, ${ }^{2} \mathrm{M}$ Davies, ${ }^{3} \mathrm{M}$ Morgan, ${ }^{4} \mathrm{~A}$ Maw, ${ }^{5} \mathrm{~N}$ Williams, ${ }^{6} \mathrm{G}$ Tudor, ${ }^{7} \mathrm{H}$ Heard, ${ }^{1} S$ Dolwani. 'Gastroenterology, Cardiff and Vale UHB, Cardiff, UK; ${ }^{2}$ Surgery, Cardiff and Vale UHB, Cardiff, UK; ${ }^{3}$ Histopathology, Cardiff and Vale UHB, Cardiff, UK; ${ }^{4}$ Surgery, Betsi Cadwalader LHB, North Wales; ${ }^{5}$ Histopathology, ABM University Health Board, Wales, UK; ${ }^{6}$ Surgery, ABM University Health Board, Wales, UK; ${ }^{7}$ Public Health, Bowel Screening Wales, Llantrisant, UK

\subsection{6/gutjnl-2014-307263.511}

Introduction Initial data from the UK BCSP demonstrated variation in referrals to surgery and management for complex polyps encountered in colonoscopy. Associated factors included local availability of operator skills and expertise with endoscopic resection, lack of a structure for discussion and standardised management at the Local assessment centres (LAC). Inappropriately high

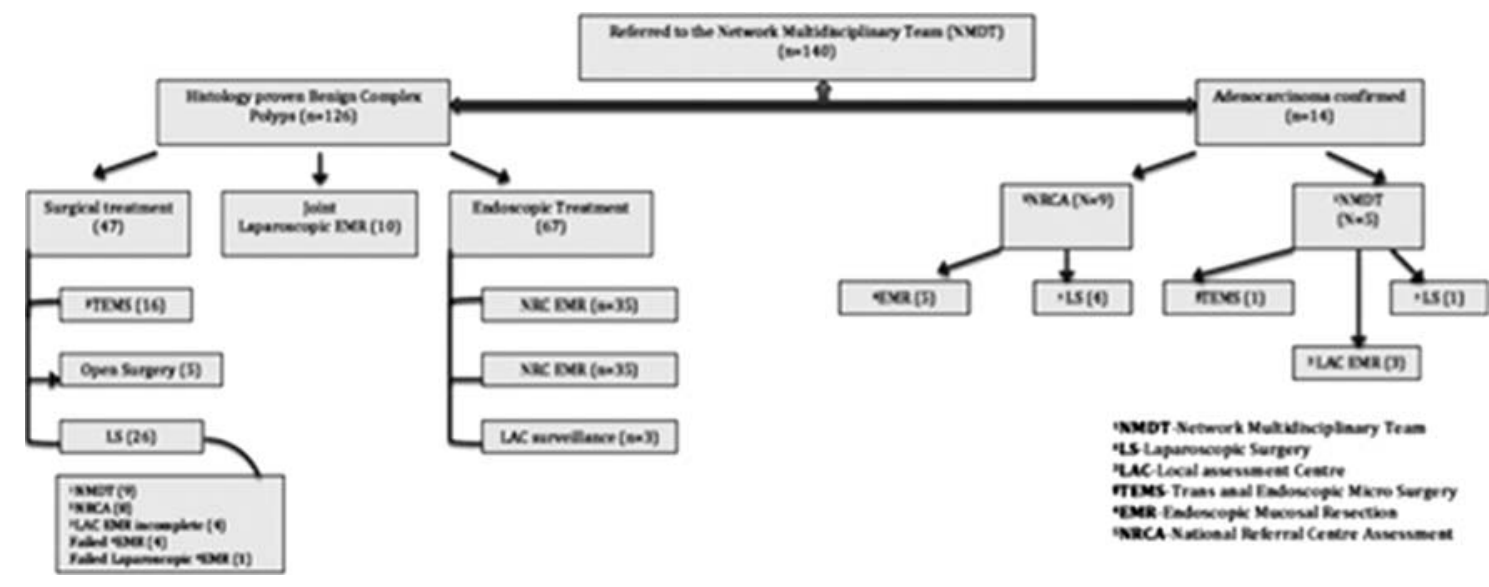

Abstract PTH-065 Figure 1 Management outcomes for complex colon polyps following referral to the NMDT 